
2 Research Square
Preprints are preliminary reports that have not undergone peer review.
They should not be considered conclusive, used to inform clinical practice, or referenced by the media as validated information.

\title{
The Prognosis and Clinicopathological Features of Different Distant Metastases Pattern in Renal Cell Carcinoma: Analysis Based on SEER Database
}

\author{
Haibin Wei \\ Zhejiang Provincial People's Hospital \\ Jia Miao \\ Zhejiang Provincial People's Hospital \\ Jianxin Cui \\ Zhejiang Provincial People's Hospital \\ Wei Zheng \\ Zhejiang Provincial People's Hospital \\ Xinpeng Chen \\ Zhejiang Provincial People's Hospital \\ Qi Zhang \\ Zhejiang Provincial People's Hospital \\ Feng Liu \\ Zhejiang Provincial People's Hospital \\ Zujie Mao \\ Zhejiang Provincial People's Hospital \\ Songlin Qiu \\ School of life science, Jiangsu university \\ Dahong Zhang ( $\sim$ zhangdahong88@yeah.net) \\ Zhejiang Provincial People's Hospital
}

\section{Research article}

Keywords: primary renal cell carcinoma, distant metastases, prognosis, SEER database, number of metastases

Posted Date: December 1st, 2020

DOI: https://doi.org/10.21203/rs.3.rs-113900/v1

License: (c) (1) This work is licensed under a Creative Commons Attribution 4.0 International License. Read Full License 


\section{Abstract}

Background: Existing data on the prognosis and clinicopathological features of patients with metastatic renal cell carcinoma (mRCC) are limited. This study aims to investigate the prognostic value and clinicopathological features of different metastatic sites in patients with mRCC.

Methods: A dataset from the National Cancer Institute's Surveillance, Epidemiology, and End Results (SEER) 18 Registries (1973-2015) was selected for a retrospective $\mathrm{mRCC}$ cohort study. There was information on distribution of metastatic lesions in lung, bone, liver, and brain in the SEER database. KaplanMeier analysis and nomogram analyses were applied to compare the survival distribution of cases. Univariate and multivariate cox regression models were used to analyze survival outcomes and risk factors.

Results: From the SEER database, a total of 10410 patients with primary mRCC from 2010 to 2015 were enrolled in this cohort study. Based on the initial metastatic sites, $54.9 \%, 37.7 \%, 19.5 \%$, and $10.4 \%$ of patients were found to have lung, bone, liver, and brain metastasis, respectively. Patients who had a single site of distant metastases accounted for 50.6\% (5268/10410). Sarcomatoid RCC had significantly higher risk to develop liver metastasis than clear cell RCC, and liver metastasis had the worst prognosis in all the patients. The median survival for patients with lung, bone, liver, and brain metastasis was 7 months, 7 months, 4 months and 5 months, respectively. The nomogram model was constructed to visually present the 1-, 3-and 5-year survival rates of patients.

Conclusion: In conclusion, different metastatic sites possess various clinicopathological features and prognostic values. Understanding these differences may contribute to designing targeted pre-treatment assessment of primary $\mathrm{mRCC}$ and creating a personalized curative intervention.

\section{Introduction}

With the increase in axial cross-sectional imaging, the incidence of kidney cancer has continued to rise over the past three decades [1,2]. New cases $(73,750)$ of kidney and renal pelvis cancer cases are estimated to be diagnosed according to Cancer statistics in 2020 , which constitutes about $4.1 \%$ of all new cancer cases in the United States [3]. It is also estimated to be responsible for 14,830 deaths (about $2.4 \%$ of all cancer deaths) [3]. About $80-85 \%$ of all primary kidney neoplasms, specifically those which originate from the renal cortex, are clear cell renal cell carcinoma (RCC) $[4,5]$. In this population, a number of patients (12-16\%) are suffering from distant metastasis at diagnosis [3,5].

Since 2010, the Surveillance, Epidemiology and End Results (SEER) data has been providing metastatic patterns including lung, bone, liver, and brain [6]. Lung metastasis are the most common site of metastatic disease and have been reported in 45\% of patients with metastatic renal cell carcinoma (mRCC) [7-9]. Bone metastasis by RCC is rare, occurring in only 3.29\% at initial diagnosis, but accounts for one-third of patients with mRCC [10-12]. Patients with liver metastasis have dismal survival outcomes and present in $23.6 \%$ of cases of newly diagnosed mRCC [13, 14]. 2.4\% of non-metastatic RCC patients later developed brain metastasis even though the incidence proportions of brain metastasis at diagnosis was $6.5 \%$ [11, 15]. Due to limited sample size, the incidence rate of metastasis to above sites may not be estimated sufficiently, and some reports on distant metastasis from RCC are just case reports. Further, few studies have focused on the distribution and overall survival (OS) of patients with $\mathrm{mRCC}$, and clinicopathological features have been less involved.

Based on the lack of knowledge of clinicopathological features on disease characteristics, we examined the association between clinicopathology and the distribution of metastatic sites in patients with RCC. The objective of this manuscript is to build on previous work by using a larger modern population-based dataset to provide guidance regarding the prognosis and clinicopathological features of patients with mRCC.

\section{Materials And Methods Database}

Our retrospective study was based on the National Cancer Institute's SEER database, a program that gathers information on cancer incidence and survival since 1973 and contains almost $28 \%$ of the population in the United States. The 18 population-based cancer registries were selected in the SEER database. 10410 patients with microscopically confirmed diagnosis of mRCC included from 2010 to 2015, since metastatic information regarding liver, lung, bone and brain was collected after 2010. Besides, other inclusion criteria were as follows: (1) all patients were staged IV using the 7th edition of the Derived American Joint Committee on Cancer (AJCC) staging system; (2) all patients were active follow-up and confirmed diagnostic age; (3) the included patients had specific metastatic details of bone, brain, liver, and lung. Exclusion criteria include tumor behavior of benign and borderline, unknown age and incomplete survival months.

\section{Outcome variables}

Some variables included to analysis selected as follows: diagnosis of age, race, gender, Fuhrman grade, TNM classification system (AJCC, 7th edition, 2010), pathological type, insurance status, married status and metastatic sites and survival months.

There are five categories of Fuhrman grade: well differentiated (Grade I), moderately differentiated (Grade II), poorly differentiated (Grade III), undifferentiated (Grade IV), and unknown.

We classified the race into 4 groups: "White", "Black", "Other" and "Unknown”.

Based on the International Classification of Diseases for Oncology, 3rd Edition (ICD-0-3) morphology codes, we identified 5 the highest frequency RCC histological type: clear cell RCC, papillary RCC, chromophobe RCC, sarcomatoid RCC and collecting duct RCC. 
As for insurance status, we reclassified patients into "Insured groups" and "Uninsured groups". Cases in "Any Medicaid" "Insured," and "Insured/No specifics" groups were collapsed into one group named "Insured groups".

Married status was defined as married and unmarried. Patients in "Single", "Separated/divorced", and "Widowed" were clustered together as "Unmarried group". Because of the confusion of the group of "Unmarried or domestic partner", we did not include it in the analysis.

The resulting data on survival status, survival time and causes for death was extricated from the database.

\section{Statistical analysis}

Descriptive statistics was utilized to sum up the patients' demographic and tumor characteristics. Chi square test was used to compare the categorical variables, and continuous variables was compared with Student's t test. Univariate and multivariable logistic regression analyses were implemented to look for a statistical relation between each independent variable and survival. Only the variables with significance in univariate analysis can be considered in the multivariate analysis. The hazard ratio (HR) and $95 \%$ confidence interval $(\mathrm{Cl})$ were utilized to assess the independent risk factors of $\mathrm{mRCC}$ in Cox proportional hazards regression model. All statistical tests were two-sides and $\mathrm{P}$ value $<0.05$ was regarded as significant statistically. The above analyses were processed on SPSS 25.0 software package (IBM Corporation, Armonk, NY, USA).

\section{Results}

Patients' characteristics

Overall, there were 10410 patients who met the screening criteria between 2010 and 2015 based SEER database, of which 7161 (68.8\%) were male, and 3249 (31.2\%) were female. The median age of all patients was 64 years old. As for TNM stage, 1831 (17.6\%) patients in T4 stage and 2301(30.7\%) in N1 stage. There were 5713 (54.9\%) patients with lung metastasis, 3920 (37.7\%) patients with bone metastasis, 2034 (19.5\%) patients with liver metastasis, and 1079 (10.4\%) patients with brain metastasis. Patients with only one site occupied 50.6\% (5268/10410) of RCC stage IV. Detailed clinical features of mRCC patients were displayed in Table1.

Table 1 Clinical features and metastasis sites for RCC 


\begin{tabular}{|c|c|c|c|c|c|c|c|c|c|c|c|}
\hline & \multicolumn{3}{|c|}{ Lung metastasis (\%) } & \multicolumn{3}{|c|}{ Bone metastasis (\%) } & \multicolumn{3}{|c|}{ Liver metastasis (\%) } & \multicolumn{2}{|c|}{ Brain metastasis (? } \\
\hline & Yes & No & $\mathrm{P}$ & Yes & No & $\begin{array}{l}\mathrm{P} \\
\text { Value }\end{array}$ & Yes & No & $\begin{array}{l}\mathrm{P} \\
\text { Value }\end{array}$ & Yes & No \\
\hline $\begin{array}{l}\text { Age at } \\
\text { diagnosis }\end{array}$ & $\begin{array}{l}63.7 \pm \\
11.7\end{array}$ & $\begin{array}{l}65.0 \pm \\
12.2\end{array}$ & $<0.001$ & $64.3 \pm 11.9$ & $\begin{array}{l}64.2 \pm \\
12.0\end{array}$ & 0.938 & $\begin{array}{l}64.0 \pm \\
12.2\end{array}$ & $\begin{array}{l}64.3 \pm \\
11.9\end{array}$ & 0.621 & $\begin{array}{l}61.8 \pm \\
10.3\end{array}$ & $\begin{array}{l}64.5 \\
12.1\end{array}$ \\
\hline Race & & & $<0.001$ & & & 0.044 & & & $<0.001$ & & \\
\hline White & $4690(54.8)$ & $3861(45.2)$ & & $3257(38.1)$ & $5294(61.9)$ & & 1598(18.7) & 6953(81.3) & & $924(10.8)$ & $762-$ \\
\hline Black & $554(50.4)$ & $546(49.6)$ & & $402(36.5)$ & $698(63.5)$ & & $292(26.5)$ & $808(73.5)$ & & $72(6.5)$ & $102 \varepsilon$ \\
\hline Other & $455(62.2)$ & $276(37.8)$ & & $256(35.0)$ & $475(65.0)$ & & 139(19.0) & $592(81.0)$ & & $82(11.2)$ & 649( \\
\hline Unknown & $14(50.0)$ & $14(50.0)$ & & $5(17.9)$ & 23(82.1) & & $5(17.9)$ & 23(82.1) & & 1(3.6) & $27(9$ \\
\hline Gender & & & 0.002 & & & 0.102 & & & $<0.001$ & & \\
\hline Male & $4002(55.9)$ & $3159(44.1)$ & & 2734(38.2) & $4427(61.8)$ & & $1321(18.4)$ & $5840(81.6)$ & & 736(10.3) & 6425 \\
\hline Female & $1711(52.7)$ & 1538(47.3) & & $1186(36.5)$ & $2063(63.5)$ & & $713(21.9)$ & $2536(78.1)$ & & $343(10.6)$ & $290 \epsilon$ \\
\hline Grade & & & 0.100 & & & $<0.001$ & & & $<0.001$ & & \\
\hline Well & $91(51.7)$ & $85(48.3)$ & & 63(35.8) & $113(64.2)$ & & $25(14.2)$ & $151(85.8)$ & & 16(9.1) & 160( \\
\hline Moderately & $496(51.1)$ & $474(48.9)$ & & 297(30.6) & 673(69.4) & & $118(12.2)$ & $852(87.8)$ & & $98(10.1)$ & 872( \\
\hline Poorly & $1185(54.5)$ & $989(45.5)$ & & $680(31.3)$ & $1494(68.7)$ & & $341(15.7)$ & 1833(84.3) & & $180(8.3)$ & 1994 \\
\hline Undifferentiated & $902(55.7)$ & 718(44.3) & & $415(25.6)$ & $1205(74.4)$ & & $243(15.0)$ & $1377(85.0)$ & & $129(8.0)$ & 1491 \\
\hline Unknown & $3039(55.6)$ & $2431(44.4)$ & & $2465(45.1)$ & $3005(54.9)$ & & 1307(23.9) & $4163(76.1)$ & & $656(12.0)$ & $481<$ \\
\hline Histology & & & $<0.001$ & & & 0.042 & & & 0.001 & & \\
\hline Clear cell RCC & $\begin{array}{l}2615 \\
(57.0)\end{array}$ & $\begin{array}{l}1970 \\
(43.0)\end{array}$ & & $\begin{array}{l}1625 \\
(35.4)\end{array}$ & $\begin{array}{l}2960 \\
(64.6)\end{array}$ & & $671(14.6)$ & $\begin{array}{l}3914 \\
(85.4)\end{array}$ & & $503(11.0)$ & $\begin{array}{l}4082 \\
(89 . c\end{array}$ \\
\hline Papillary RCC & $216(41.6)$ & $303(58.4)$ & & $157(30.3)$ & $362(69.7)$ & & $86(16.6)$ & $433(83.4)$ & & $30(5.8)$ & 489 \\
\hline $\begin{array}{l}\text { Chromophobe } \\
\text { RCC }\end{array}$ & $29(34.5)$ & $55(65.5)$ & & $29(34.5)$ & $55(65.5)$ & & $16(19.0)$ & $68(81.0)$ & & $6(7.1)$ & $78(\mathrm{c}$ \\
\hline $\begin{array}{l}\text { Sarcomatoid } \\
\text { RCC }\end{array}$ & $311(57.1)$ & $234(42.9)$ & & $210(38.5)$ & $335(61.5)$ & & $117(21.5)$ & $428(78.5)$ & & $46(8.4)$ & 499 \\
\hline $\begin{array}{l}\text { Collecting duct } \\
\text { RCC }\end{array}$ & $42(63.6)$ & $24(36.4)$ & & $28(42.4)$ & $38(57.6)$ & & $13(19.7)$ & $53(80.3)$ & & $2(3.0)$ & $64(c$ \\
\hline T stage & & & $<0.001$ & & & $<0.001$ & & & $<0.001$ & & \\
\hline $\mathrm{T} 1$ & $732(42.9)$ & $974(57.1)$ & & $927(54.3)$ & $779(45.7)$ & & $265(15.5)$ & $1441(84.5)$ & & $165(9.7)$ & 1541 \\
\hline T2 & $994(64.1)$ & $556(35.9)$ & & 619(39.9) & $931(60.1)$ & & $279(18.0)$ & $1271(82.0)$ & & 259(16.7) & 1291 \\
\hline T3 & $2143(64.4)$ & 1186(35.6) & & 1059(31.8) & $2270(68.2)$ & & $579(17.4)$ & $2750(82.6)$ & & $311(9.3)$ & $301 \varepsilon$ \\
\hline $\mathrm{T} 4$ & $815(44.5)$ & $1016(55.5)$ & & $392(21.4)$ & $1439(78.6)$ & & $439(24.0)$ & $1392(76.0)$ & & $121(6.6)$ & $171 c$ \\
\hline Unknown & $988(52.6)$ & $890(47.4)$ & & $864(46.0)$ & $1014(54.0)$ & & $452(24.1)$ & $1426(75.9)$ & & $217(11.6)$ & 1661 \\
\hline N stage & & & $<0.001$ & & & $<0.001$ & & & $<0.001$ & & \\
\hline NO & $3115(53.1)$ & $2755(46.9)$ & & $2169(37.0)$ & $3701(63.0)$ & & $931(15.9)$ & $4939(84.1)$ & & $639(10.9)$ & 5231 \\
\hline N1 & $1869(58.4)$ & 1332(41.6) & & 1179(36.8) & $2022(63.2)$ & & 797(24.9) & $2404(75.1)$ & & 293(9.2) & $290 \varepsilon$ \\
\hline Unknown & $729(54.4)$ & $610(45.6)$ & & $572(42.7)$ & $767(57.3)$ & & $306(22.9)$ & 1033(77.1) & & $147(11.0)$ & 1192 \\
\hline Insurance & & & $<0.001$ & & & 0.384 & & & 0.633 & & \\
\hline Insured & $5414(55.2)$ & $4391(44.8)$ & & $3696(37.7)$ & $6109(62.3)$ & & $1909(19.5)$ & 7896(80.5) & & 1012(10.3) & $879=$ \\
\hline Uninsured & $237(57.5)$ & $175(42.5)$ & & 160(38.8) & $252(61.2)$ & & $88(21.4)$ & $324(78.6)$ & & $58(14.1)$ & 354( \\
\hline Unknown & $62(32.1)$ & $131(67.9)$ & & $64(33.2)$ & $129(66.8)$ & & $37(19.2)$ & 156(80.8) & & $9(4.7)$ & 184( \\
\hline Married status & & & $<0.001$ & & & 0.079 & & & 0.025 & & \\
\hline Married & $3382(56.3)$ & $2628(43.7)$ & & 2238(37.2) & $3772(62.8)$ & & $1125(18.7)$ & $4885(81.3)$ & & $628(10.4)$ & 5382 \\
\hline Unmarried & 2095(53.8) & $1801(46.2)$ & & 1511(38.8) & 2385(61.2) & & $814(20.9)$ & $3082(79.1)$ & & $418(10.7)$ & $347 \varepsilon$ \\
\hline Unknown & $223(46.2)$ & $260(53.8)$ & & 165(34.2) & $318(65.8)$ & & $90(18.6)$ & $393(81.4)$ & & $31(6.4)$ & 452( \\
\hline
\end{tabular}


Abbreviations: RCC, renal cell carcinoma

Lung metastasis

Lung is the most common site of synchronous metastasis for mRCC patients among the cohort with metastatic disease. The mean age of cases without lung metastasis was 1.3 years elder than cases diagnosed with. White patients had higher proportion of lung metastasis than others in the ethnicity of patients. As compared to females, a larger percentage of males were lung metastasis. T3 patients had the highest rate of lung metastasis, and T1 had the lowest rate in the classification of T stage. For N stage classification, N1 had significantly higher rate of lung metastasis than N0, $58.4 \%$ vs. $53.1 \%, \mathrm{P}<0.001$. Married and uninsured patients had a significantly higher than other types in metastatic rate specifically, both $\mathrm{P}<0.001$. Fuhrman grade did not show significant difference in lung metastasis.

Bone metastasis

As for bone metastasis, there was no difference between male and female patients. The same phenomenon occurred in diagnostic age, insurance status, and married status. White patients had higher percentages of bone metastasis than black patients and other races. T1 patients accounted for the largest proportion in T stage classification in bone metastasis. Unlike the results of lung metastasis, NO had higher percentages of bone metastasis. For Fuhrman grade, undifferentiated (grade IV) presented the lowest rate.

Liver metastasis

There are many different results in liver metastasis compared to bone and lung metastasis. For ethnicity, black patients had a higher percentage than other races, which did not happen in above metastasis. Regardless of gender and married status, the opposite results were shown in liver metastasis, as females were much more than males and unmarried patients were more than married. Patients in T4 were the most common types in T stage classification in liver metastasis. In terms of N stage classification, patients in N1 had higher liver metastasis than other kinds. Insurance state and diagnostic age showed no statistically significant difference.

Brain metastasis

Some features for patients with brain metastasis resemble those with lung metastasis, involving age at diagnosis, ethnicity, and T stage classification. N0 patients presented significant difference with $\mathrm{N} 1$ in brain metastasis. Unexpectedly, patients with uninsured and unmarried had higher percentage than those insured and married, both $\mathrm{P}<0.05$. Undifferentiated differentiated tumors had lower brain metastatic rate than well, moderately, and poorly tumors. There was no significant difference between males and females.

Combination of metastases

There were many patients suffered more than one metastasis. Except for one-site metastasis, 11 combinations of metastases were found in Table 2. As shown in Figure 1, Venn diagram was used to illustrate the distribution of the mRCC patients. The patients of bone and lung metastasis were the highest frequency in the two-site metastasis, which achieving to $10.82 \%(1126 / 10410)$. Only 12 patients had bone and brain and liver metastasis, which was the least common in metastatic mRCC. There were still 91 patients had all four sites metastasis.

Table 2 Frequencies of combination metastasis and 3, 5-y OS 


\begin{tabular}{|lllll|}
\hline & Number (\%) & 3-y OS & 5 -y OS & Median OS (mo) \\
\hline One site & & & & \\
\hline Only Lung & $2796(26.86)$ & $9.05 \%$ & $1.54 \%$ & 11 \\
\hline Only Bone & $1719(16.51)$ & $10.99 \%$ & $2.27 \%$ & 12 \\
\hline Only Liver & $534(5.13)$ & $6.74 \%$ & $0.75 \%$ & 5 \\
\hline Only Brain & $219(2.10)$ & $7.76 \%$ & $1.37 \%$ & 9 \\
\hline Two sites & & & & \\
\hline Bone and brain & $97(0.93)$ & $2.06 \%$ & 0 & 5 \\
\hline Bone and liver & $232(2.22)$ & $3.45 \%$ & 0 & 4 \\
\hline Bone and lung & $1126(10.82)$ & $4.62 \%$ & $0.44 \%$ & 6 \\
\hline Brain and liver & $23(0.22)$ & 0 & 0 & 3 \\
\hline Brain and lung & $337(3.24)$ & $3.56 \%$ & $0.30 \%$ & 6 \\
\hline Liver and lung & $641(6.16)$ & $2.18 \%$ & $0.16 \%$ & 4 \\
\hline Three sites & & & & 3 \\
\hline Bone and brain and liver & $12(0.12)$ & 0 & 0 & 3 \\
\hline Bone and brain and lung & $221(2.12)$ & $1.81 \%$ & 0 & 4 \\
\hline Bone and liver and lung & $422(4.05)$ & $1.81 \%$ & 0 & 3 \\
\hline Brain and liver and lung & $79(0.76)$ & $1.27 \%$ & 0 & 3 \\
\hline Four sites & $91(0.87)$ & 0 & 0 & 3 \\
\hline Bone and brain and liver and lung & & & & \\
\hline
\end{tabular}

Abbreviations: OS: overall survival

Pathological distribution

Patients were grouped by the most frequent pathotype in SEER database, the distribution of distant metastases were shown in Figure 2. In terms of the type of clear cell RCC and chromophobe RCC, the percentage of exclusive lung metastasis was higher to lung and other metastases. The Same phenomenon of clear cell RCC were reappeared in bone and liver metastasis. It is interesting that liver metastasis showed differences between pathological types. Except for clear cell RCC, the liver plus other metastases possessed higher percentage than exclusive liver (Figure 2c). As expected, brain and other metastases were much more than exclusive brain metastasis, and it had no statistical significance between pathological types (Figure 2d).

Univariate survival analysis of distant metastases sites

In Table 3, we took the variables included the metastatic site, ethnicity, gender, grade, T stage, N stage, insurance status, and married status. All the variables were regarded as prognostic factors relating to overall survival apart from insurance status. Liver metastasis informed the worst OS in one-site metastasis of four metastatic sites. Among the cohort, bone metastasis presented the minimum hazard ratio (HR). Black patients exhibited worse prognosis with white patients in OS $(P<0.001)$. Compared to males, there were worse prognosis in females. The interesting phenomenon was observed in Fuhrman grade that patients with moderately differentiated grade appeared better OS than well differentiated grade $(P=0.003)$. Similarly, patients of T3 seemed to exhibit survival advantage compared to patients of T1. As we expected, patients of N1 had worse prognosis than those of N0. As for histology, Sarcomatoid RCC owned the worst OS than the RCC originated from epithelium. The significant difference in married status was observed, the unmarried patients were prone to worse outcome. We utilized Kaplan-Meier analysis to create survival curves among the patients with single metastasis and two-site metastasis (Figure3a, 3b). As for the patients with three-site metastasis, the log-rank tests showed that no significant difference between them (Figure3c).

Table 3 Univariate survival analysis of patients with four single metastases 


\begin{tabular}{|c|c|c|}
\hline \multirow[t]{2}{*}{ Characteristics } & \multicolumn{2}{|l|}{ Overall survival } \\
\hline & $\mathrm{HR}(95 \% \mathrm{Cl})$ & $\mathrm{p}$-value \\
\hline Metastatic site & & $<0.001$ \\
\hline Lung & 1 & Ref. \\
\hline Bone & $0.969(0.901-1.042)$ & 0.393 \\
\hline Liver & $1.490(1.341-1.655)$ & $<0.001$ \\
\hline Brain & 1.118 (0.948-1.319) & 0.185 \\
\hline Race & & $<0.001$ \\
\hline White & 1 & Ref. \\
\hline Black & $1.151(1.071-1.238)$ & $<0.001$ \\
\hline Other & $0.930(0.850-1.018)$ & 0.115 \\
\hline Gender & & 0.020 \\
\hline Male & 1 & Ref. \\
\hline Female & $1.060(1.009-1.113)$ & 0.020 \\
\hline Grade & & $<0.001$ \\
\hline Well & 1 & Ref. \\
\hline Moderate & $0.734(0.597-0.903)$ & 0.003 \\
\hline Poorly & $1.076(0.886-1.308)$ & 0.460 \\
\hline Undifferentiated & $1.358(1.115-1.653)$ & 0.002 \\
\hline Histology & & $<0.001$ \\
\hline Clear cell RCC & 1 & Ref. \\
\hline Papillary RCC & $1.327(1.188-1.482)$ & $<0.001$ \\
\hline Chromophobe RCC & $0.896(0.679-1.181)$ & 0.435 \\
\hline Sarcomatoid RCC & $2.513(2.273-2.778)$ & $<0.001$ \\
\hline Collecting duct RCC & $2.110(1.607-2.770)$ & $<0.001$ \\
\hline T stage & & $<0.001$ \\
\hline $\mathrm{T} 1$ & 1 & Ref. \\
\hline T2 & $1.092(1.005-1.186)$ & 0.038 \\
\hline T3 & $0.905(0.843-0.972)$ & 0.006 \\
\hline T4 & $1.222(1.130-1.322)$ & $<0.001$ \\
\hline N stage & & $<0.001$ \\
\hline NO & 1 & Ref. \\
\hline N1 & $1.770(1.682-1.862)$ & $<0.001$ \\
\hline Insurance status & & 0.111 \\
\hline Insured & 1 & Ref. \\
\hline Uninsured & $1.096(0.979-1.228)$ & 0.111 \\
\hline Married status & & $<0.001$ \\
\hline Married & 1 & Ref. \\
\hline Unmarried & $1.241(1.184-1.301)$ & $<0.001$ \\
\hline
\end{tabular}

Abbreviations: $\mathrm{HR}$, hazard ratio; $\mathrm{Cl}$, confidence interval; Ref, reference; RCC: renal cell carcinoma Multivariate survival analysis of distant metastases sites

On multivariable Cox regression, ethnicity, gender and married status were not the independent factors for mRCC $(P>0.05)$. As for metastatic site, liver metastasis was still the worst prognostic metastasis. Meanwhile, the interesting situation that moderately differentiated grade with better OS was reappeared 
on multivariate survival analysis. Sarcomatoid RCC had worse outcomes than other histological types as well. The anticipated event happened again when including the T stage on multivariate survival analysis that the patients of T3 showed better survival than patients with T1. Regional lymph nodes negative was the positive factor for patients with IV stage. (Table 4).

Table 4 Multivariate survival analysis of patients with four single metastases

\begin{tabular}{|c|c|c|}
\hline \multirow[t]{2}{*}{ Characteristics } & \multicolumn{2}{|l|}{ Overall survival } \\
\hline & $\mathrm{HR}(95 \% \mathrm{Cl})$ & $\mathrm{p}$-value \\
\hline \multicolumn{3}{|l|}{ Metastasis site } \\
\hline Lung & 1 & Ref. \\
\hline Bone & $0.896(0.765-1.050)$ & 0.175 \\
\hline Liver & $1.201(0.932-1.546)$ & 0.156 \\
\hline Brain & $1.213(0.878-1.675)$ & 0.242 \\
\hline \multicolumn{3}{|l|}{ Race } \\
\hline White & 1 & Ref. \\
\hline Black & $0.862(0.661-1.124)$ & 0.273 \\
\hline Other & $0.827(0.649-1.052)$ & 0.124 \\
\hline \multicolumn{3}{|l|}{ Gender } \\
\hline Male & 1 & Ref. \\
\hline Female & $1.052(0.911-1.214)$ & 0.489 \\
\hline \multicolumn{3}{|l|}{ Grade } \\
\hline Well & 1 & Ref. \\
\hline Moderate & $0.934(0.594-1.470)$ & 0.769 \\
\hline Poorly & $1.109(0.709-1.733)$ & 0.651 \\
\hline Undifferentiated & $1.335(0.848-2.102)$ & 0.212 \\
\hline \multicolumn{3}{|l|}{ Histology } \\
\hline Clear cell RCC & 1 & Ref. \\
\hline Papillary RCC & $1.509(1.159-1.964)$ & 0.002 \\
\hline Chromophobe RCC & $0.968(0.567-1.652)$ & 0.905 \\
\hline Sarcomatoid RCC & $2.072(1.662-2.585)$ & $<0.001$ \\
\hline Collecting duct RCC & $2.457(1.429-4.225)$ & 0.001 \\
\hline \multicolumn{3}{|l|}{ T stage } \\
\hline $\mathrm{T} 1$ & 1 & Ref. \\
\hline $\mathrm{T} 2$ & $1.061(0.833-1.351)$ & 0.634 \\
\hline T3 & $1.125(0.915-0.382)$ & 0.264 \\
\hline T4 & $1.745(1.345-2.265)$ & $<0.001$ \\
\hline \multicolumn{3}{|l|}{ N stage } \\
\hline No & 1 & Ref. \\
\hline N1 & $1.749(1.514-2.021)$ & $<0.001$ \\
\hline \multicolumn{3}{|l|}{ Married status } \\
\hline Married & 1 & Ref. \\
\hline Unmarried & $1.145(0.998-1.314)$ & 0.054 \\
\hline
\end{tabular}

Abbreviations: HR, hazard ratio; $\mathrm{Cl}$, confidence interval; Ref, reference; RCC: renal cell carcinoma 


\section{Construction of prognosis model for distant metastases sites}

Meaningful factors were selected for the nomogram model construction relied on the multivariate survival analysis and clinical availability. The included factors were as follow: age, grade, $\mathrm{T} / \mathrm{N}$ stage, histology and distant metastases sites. Every factor had its score corresponding to the points at the top of the nomogram. For instance, in N stage, the N0 scores 0 points and the N1 scores 32 points. 1-year survival rate, 3-year survival rate and 5-year survival rate were acquired based on the commensurate points, respectively. If the total points is 160 , the 1 -year survival rate is $52 \%$, the 3 -year survival rate is $20 \%$ and the 5 year survival rate is $10 \%$ (Figure4)

\section{Discussion}

Within the current study, we identified 10410 individuals with mRCC between years 2010 and 2015 . The number of patients enrolled was significantly more than previous literatures from the SEER database, which was 6610 for Chandrasekar and colleagues, and 5992 for and colleagues [8, 16]. In our study, the metastatic rate to lung, bone, liver, and brain was $54.9 \%, 37.7 \%, 19.5 \%$, and $10.4 \%$, respectively. The metastatic rate to above three out of four sites were similar to previous reports, which were $45.2 \%-51.2 \%$ for lung metastasis, $17.0 \%-20.3 \%$ for liver metastasis, and $8.1 \%-9.8 \%$ for brain metastasis [ 8,11 ]. The metastatic rate to bone was slightly higher than previous literature reports, which was $20 \%-33.5 \%$ for bone metastasis $[8,10]$. The fact that bone metastasis was initially underestimated is improving. This improving could be due to the following causes: 1) More attention had been paid to evaluate status of metastasis to above sites using appropriate modalities. Both NCCN and EAU recommend bone imaging in symptomatic patients or in those with an unusual alkaline phosphatase (ALP) level $[17,18]$. In the presence of an elevated ALP or clinical symptoms, the probability of a positive bone scan increases to approximately $5 \%$ to $10 \%[19]$. 2) More and more biochemical markers are emerging, and some of them will play a role in the diagnosis of bone metastasis of RCC now and in the future. The "vicious cycle" hypothesis is used to describe how RCC cells interact with the bone microenvironment to drive bone destruction and tumor growth [12]. In this process, many biomarkers and signaling pathways play a role, including TGF- $\beta$, TGF- $\alpha$ /EGF-R signaling, insulin mRNA binding protein-3 (IMP3), cadherin-11, PTHrP, calcium/CaSR, AKT/integrin-a5 signaling, matriptase, MET and miRNAs. Klepzig found that procollagen type 1 aminoterminal propeptide (P1NP) concentration was significantly higher among those with bone metastasis than in those without 20]. It suggests that P1NP may be a significant early predictor for RCC bone metastasis and may play a certain role in the initial diagnosis. 3) Previous research found that lung or liver metastasis have a higher risk of bone metastasis than those without lung nor liver metastasis in colorectal cancer and gastric cancer [6, 21]. Our study showed similar phenomenon of multiple metastases in RCC. The number of combined bone metastasis was higher than that of exclusive bone metastasis, for sarcomatoid RCC, collecting duct RCC, papillary RCC, and chromophobe RCC. This insinuation is helpful for us to design screen strategy. Once the other metastases occur, bone scanning can help increase the rate of bone metastasis. Knowledge of metastatic site distribution may be helpful for clinicians to design personalized examinations for RCC patients.

The data from the current analysis indicated that chromophobe RCC and clear cell RCC has the best survival, which is similar to that found by Abdel-Rahman and colleagues [16]. The rate of metastases in a single site was $50.6 \%$ versus $49.4 \%$ in two or more sites. Compared to exclusive liver metastasis, sarcomatoid RCC, collecting duct RCC, and papillary RCC are more prone to multiple metastases. For all clinicopathological types, brain metastasis did not tend to appear alone and were more likely to be associated with other metastases. Our analysis found metastatic RCC patients have the worse survival with the increase of metastasis site. We therefore guessed that metastatic disease burden were associated with increased sites, and patients might receive less intervention time.

Our subsequent assessment of survival analysis of metastatic disease has similar results as previously reported [8, 16]. On univariate survival analysis of patients with four single metastasis at the time of diagnosis, statistically significant parameters were disease-specific factors, such as metastatic site, race, gender, grade, histology, T stage, $\mathrm{N}$ stage, and married status. Among the parameters mentioned above, metastatic site plays an important role. When specifically looking at multivariate survival analysis of patients with four single metastasis, the same factors, including metastatic site, grade, histology, $T$ stage and N stage, predicted for worse prognostic metastasis. In univariate survival analysis, our study showed that unmarried RCC patients were at worse overall survival than married status with the possibility that spouse might provide social supports and encourage the patients to seek medical treatments.

The outcome for RCC patients with metastasis was poor, which were 7 months, 7 months, 4 months, and 5 months for metastasis to lung, bone, liver, and brain metastasis, respectively. The nomogram is a convenient graphical representation of a mathematical model, in which various important factors are combined to predict a future endpoint [22]. By integrating clinical and pathological factors, the nomogram was used to provide visual estimates of the 1-, 3and 5-year survival rates of patients in the study. To date, several RCC nomograms have been generated for predicting the probability of RCC recurrence and survival [22-24]. The first nomogram was designed to calculate the likelihood of recurrence after the operation for RCC patients in 2001 by Kattan et al [22]. Sorbellini et al published a postoperative prognostic nomogram in 2005 , which was designed to predict recurrence for patients with conventional clear cell RCC [24]. Zhang et al developed a nomogram to predict the overall survival and the disease-specific survival from clear cell RCC treated with nephrectomy [23]. Due to the important effect of metastasis on prognosis, the aim of this study was to establish comprehensive and practical nomogram, based on distant metastasis sites, for predicting the survival rate of RCC patients. Meaningful factors were selected for the nomogram model construction relied on the multivariate survival analysis and clinical availability in the study.

Inevitably, there are sereval limitations due to the limited information of SEER database and the retrospective nature of the study. First of all, the metastatic data to above 4 sites were provided since 2010 and follow-up time is not very long. Secondly, comparing to those patients with synchronous metastasis, metachronous metastasis may possess larger quantity. Besides, information on other metastatic sites is bland, such as ovary, other urinary, gastrointestinal digestive system, and adrenal gland. Furthermore, the SEER is an observational retrospective database relying on ICD codes for assessment of secondary diagnostic codes, which may be subject to potential coding biases. Despite these limitations from SEER, our population represents the largest cohort used for the assessment of different site-specific mRCC. Our data are highly generalizable since it originates from a nationwide sample, and might provide some useful knowledge for predicting the clinical outcomes and guiding decisions regarding surgery, surveillance, and adjuvant therapies. 


\section{Conclusion}

In conclusion, heterogeneity exists in the oncological outcomes of patients with site-specific metastasis. Patients with bone metastasis appear to show the best oncologic survival and those with brain metastasis show the worst survival among those with single metastasis. Relying on different histological types, there are numerous metastatic features and prognostic values. Knowledge of these differences in metastatic patterns may contribute to designing targeted pre-treatment assessment of renal cell carcinoma and making a personalized curative intervention.

\section{Abbreviations}

RCC: renal cell carcinoma; SEER: Surveillance, Epidemiology, and End Results; OS: Overall survival; mRCC: metastatic renal cell carcinoma; AJCC: Derived American Joint Committee on Cancer; IDC, ILC: Infiltrating; HR: hazard ratio; Cl: confidence interval.

\section{Declarations}

\section{Ethics approval and consent to participate}

All procedures performed in studies involving human participants were in accordance with the 1964 Helsinki declaration and its later amendments or comparable ethical standards. We signed the 'Surveillance, Epidemiology, and End Results Program Data Use Agreement' in accordance with the requirement of using SEER database. Approval was waived by the local ethics committee, as SEER data is publicly available and de-identified.

\section{Consent for publication}

All authors listed approved the publication of the manuscript.

\section{Availability of data and material}

The datasets analyzed during the current study are publicly available for use in accordance with a limited use agreement for SEER research data: Surveillance, Epidemiology, and End Results (SEER) Program (https://seer.cancer.gov) SEER*Stat Database.

\section{Competing interests}

The authors declare that they have no conflict of interest.

\section{Funding}

This study was funded by Medical Scientific Research Foundation of Zhejiang Province (Grant No. 2018KY019 and 2021KY449).

\section{Authors' contributions}

HBW and DHZ designed the study. JM, JXC and SLQ contributed to data acquisition and statistical analysis and prepared the manuscript. JM, XPC, WZ and QZ drafted the manuscript. All authors revised this manuscript critically. HBW, FL, ZJM and DHZ supervised the study. All authors read and approved the final manuscript.

\section{Acknowledgments}

All authors would like to thank SEER for open access to the database

\section{Code availability}

SEER*stat 8.3.6 software was used to extract the data.

\section{References}

1. Smaldone MC, Egleston B, Hollingsworth JM, Hollenbeck BK, Miller DC, Morgan TM, et al. Understanding Treatment Disconnect and Mortality Trends in Renal Cell Carcinoma Using Tumor Registry Data. Med Care. 2017;55(4):398-404.

2. Hollenbeak CS, Schaefer EW, Doan J, Raman JD. Determinants of treatment in patients with stage IV renal cell carcinoma. BMC Urol. 2019 Nov 29;19(1):123.

3. Siegel RL, Miller KD, Jemal A. Cancer statistics, 2020. CA Cancer J Clin. 2020;70(1):7-30.

4. Rueckert J, Devitt K, Gardner JA. Renal Cell Carcinoma with monosomy 8: A Case Series and Review of the Literature. J Assoc Genet Technol. 2018;44(1):5-9.

5. Abdelaziz A, Vaishampayan U. Cabozantinib for Renal Cell Carcinoma: Current and Future Paradigms. Curr Treat Options Oncol. 2017;18(3):18.

6. Qiu MZ, Shi SM, Chen ZH, Yu HE, Sheng H, Jin Y, et al. Frequency and clinicopathological features of metastasis to liver, lung, bone, and brain from gastric cancer: A SEER-based study. Cancer Med. 2018;7(8):3662-72.

7. Lin S, Zheng Y, Qin Z, Hu X, Qi F, Yin R, et al. Surgical intervention in renal cell carcinoma patients with lung and bronchus metastasis is associated with longer survival time: a population-based analysis. Ann Transl Med. 2019;7(14):323. 
8. Chandrasekar T, Klaassen Z, Goldberg H, Kulkarni GS, Hamilton RJ, Fleshner NE. Metastatic renal cell carcinoma: Patterns and predictors of metastases-A contemporary population-based series. Urol Oncol. 2017;35(11):661.e7-661.e14.

9. de Velasco G, Wankowicz SA, Madison R, Ali SM, Norton C, Duquette A, et al. Targeted genomic landscape of metastases compared to primary tumours in clear cell metastatic renal cell carcinoma. Br J Cancer. 2018;118(9):1238-42.

10. Guo Q, Zhang C, Guo X, Tao F, Xu Y, Feng G, et al. Incidence of bone metastasis and factors contributing to its development and prognosis in newly diagnosed renal cell carcinoma: a population-based study. Cancer Manag Res. 2018 Aug 28;10:2935-2944.

11. Bianchi M, Sun M, Jeldres C, Shariat SF, Trinh QD, Briganti A, et al. Distribution of metastatic sites in renal cell carcinoma: a population-based analysis. Ann Oncol. 2012 Apr;23(4):973-80.

12. Chen SC, Kuo PL. Bone Metastasis from Renal Cell Carcinoma. Int J Mol Sci. 2016 Jun 22;17(6):987.

13. Guo B, Liu S, Wang M, Hou H, Liu M. The role of cytoreductive nephrectomy in renal cell carcinoma patients with liver metastasis. Bosn J Basic Med Sci. 2020 Jul 23. doi: 10.17305/bjbms.2020.. Epub ahead of print.

14. Kim SH, Park WS, Park B, Pak S, Chung J. A Retrospective Analysis of the Impact of Metastasectomy on Prognostic Survival According to Metastatic Organs in Patients With Metastatic Renal Cell Carcinoma. Front Oncol. 2019 May;22:9:413. doi:10.3389/fonc.2019.00413.

15. Barnholtz-Sloan JS, Sloan AE, Davis FG, Vigneau FD, Lai P, Sawaya RE. Incidence proportions of brain metastases in patients diagnosed (1973 to 2001) in the Metropolitan Detroit Cancer Surveillance System. J Clin Oncol. 2004 Jul;15(14):2865-72. 22(.

16. Abdel-Rahman O. Clinical correlates and prognostic value of different metastatic sites in metastatic renal cell carcinoma. Future Oncol. 2017 Sep;13(22):1967-80.

17. Tyson MD, Chang SS. Optimal Surveillance Strategies After Surgery for Renal Cell Carcinoma. J Natl Compr Canc Netw. 2017;15(6):835-40.

18. Ljungberg B, Bensalah K, Canfield S, Dabestani S, Hofmann F, Hora M, et al. EAU guidelines on renal cell carcinoma: 2014 update. Eur Urol. 2015 May;67(5):913-24.

19. Koga S, Tsuda S, Nishikido M, Ogawa Y, Hayashi K, Hayashi T,et al. The diagnostic value of bone scan in patients with renal cell carcinoma. J Urol. 2001 Dec;166(6):2126-8.

20. Klepzig M, Sauer-Eppel H, Jonas D, Oremek GM. Value of procollagen type 1 amino-terminal propeptide in patients with renal cell carcinoma. Anticancer Res. 2008 Jul-Aug;28(4C):2443-6.

21. Qiu M, Hu J, Yang D, Cosgrove DP, Xu R. Pattern of distant metastases in colorectal cancer: a SEER based study. Oncotarget. 2015 Nov 17;6(36):3865866.

22. Kattan MW, Reuter V, Motzer RJ, Katz J, Russo P. A postoperative prognostic nomogram for renal cell carcinoma. J Urol. 2001 Jul;166(1):63-7.

23. Zhang G, Wu Y, Zhang J, Fang Z, Liu Z, Xu Z, et al. Nomograms for predicting long-term overall survival and disease-specific survival of patients with clear cell renal cell carcinoma. Onco Targets Ther. 2018 Sep;6:11:5535-44.

24. Sorbellini M, Kattan MW, Snyder ME, Reuter V, Motzer R, Goetzl M, McKiernan J, Russo P. A postoperative prognostic nomogram predicting recurrence for patients with conventional clear cell renal cell carcinoma. J Urol. 2005 Jan;173(1):48-51.

\section{Figures}




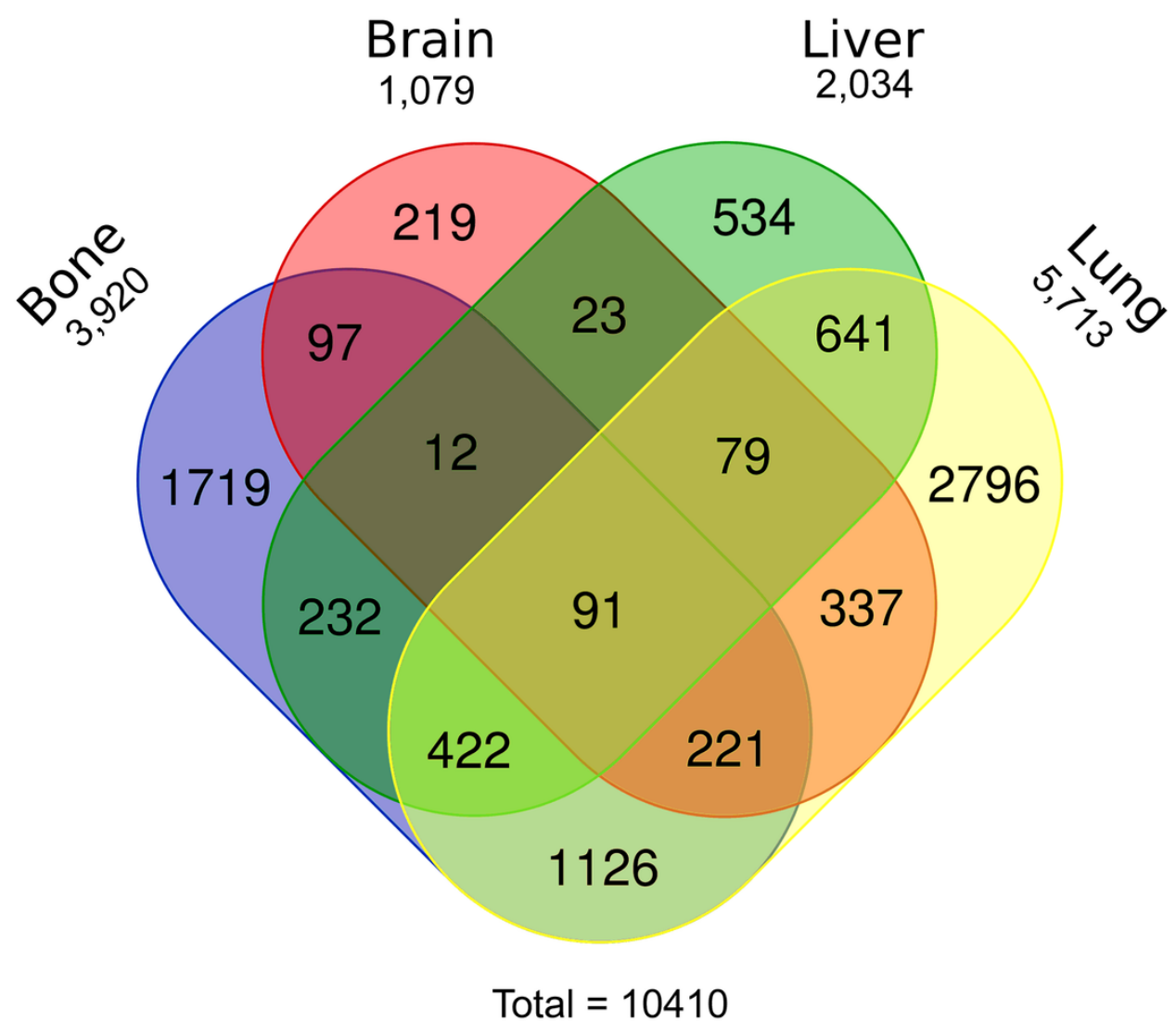

Figure 1

Venn diagram of the distribution of distant metastatic sites. There were four types of metastatic sites in 10410 patients. Lung metastasis was the most common forms of metastasis 


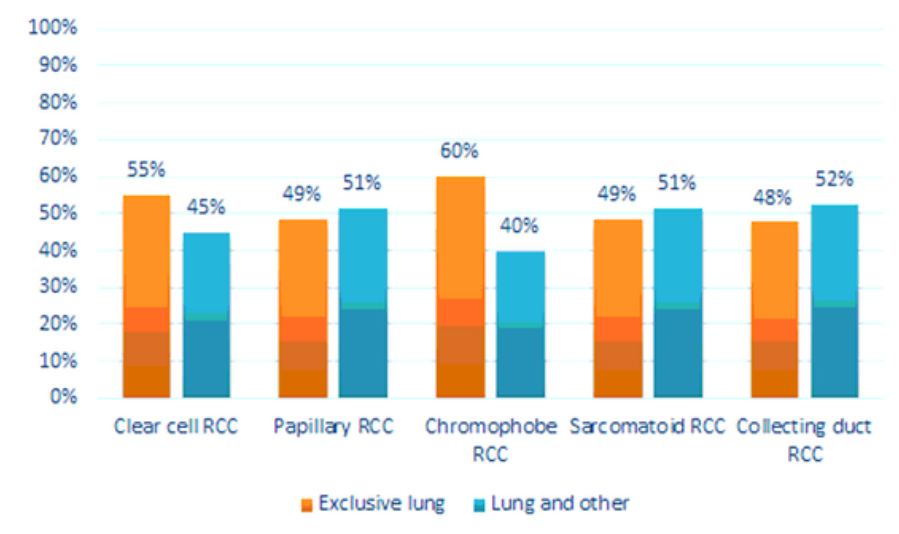

C

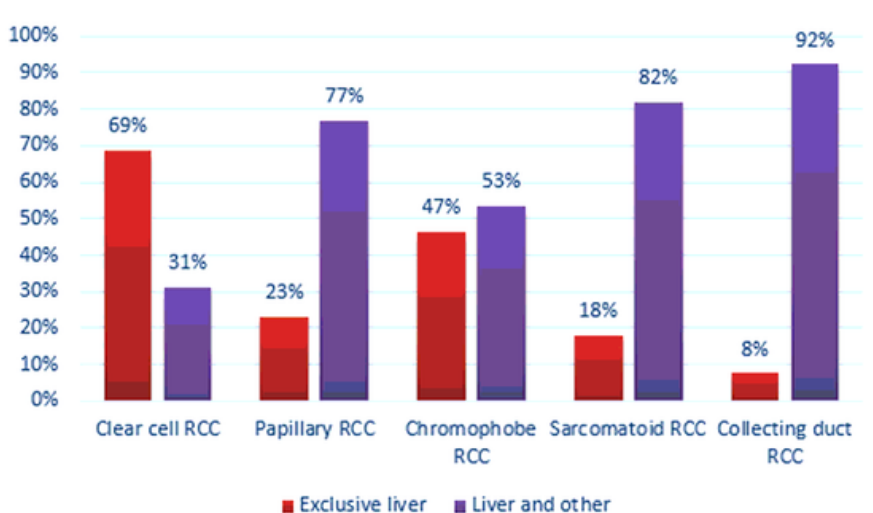

b

d

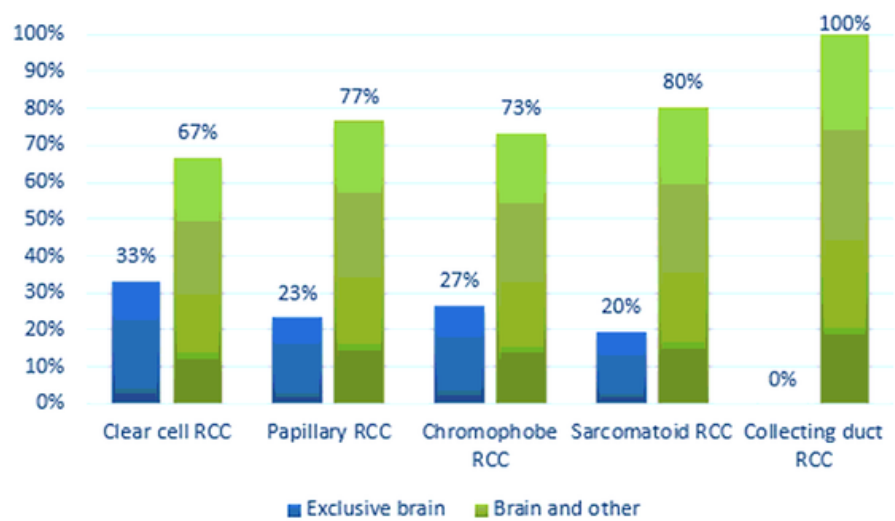

\section{Figure 2}

The distribution of metastases in a single versus multiple concomitant sites, stratified by pathology categories, in the four kinds of metastatic sites: lung (a), bone (b), liver (c), and brain (d).
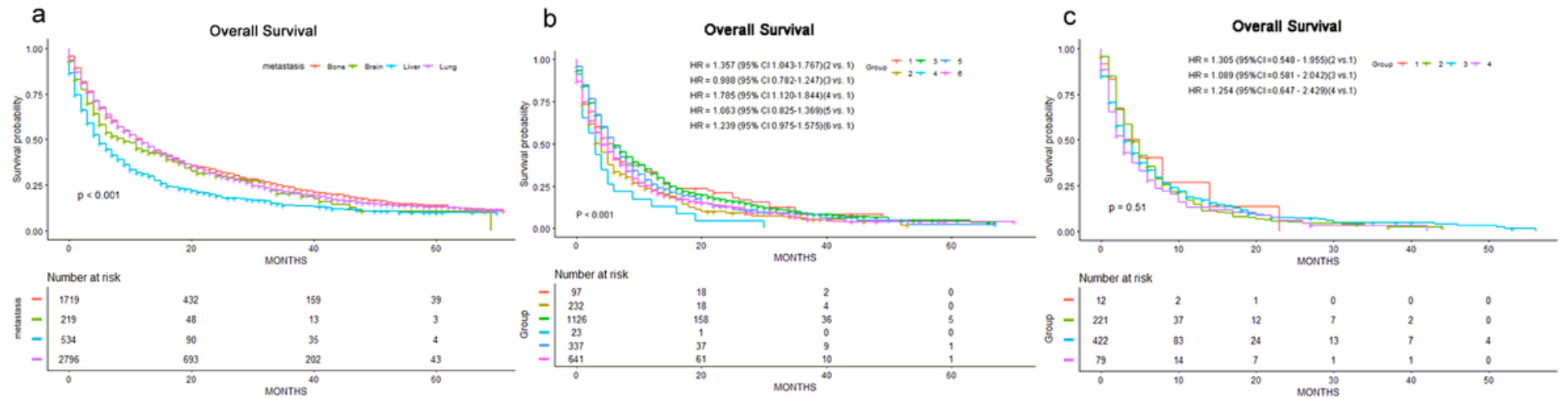

Figure 3

Kaplan-Meier curves and Log-Rank test for OS according to the number of metastasis. (a) only one site metastasis. (b) two sites metastases. 1, Bone and brain metastases; 2, Bone and liver metastases; 3, Bone and lung metastases; 4, Brain and liver metastases; 5, Brain and lung metastases; 6, Liver and lung metastases. (c) three sites metastases. 1, Bone and brain and liver metastases; 2, Bone and brain and lung metastases; 3 Bone and liver and lung metastases; 4, Brain and liver and lung metastases; HR, hazard ratio 


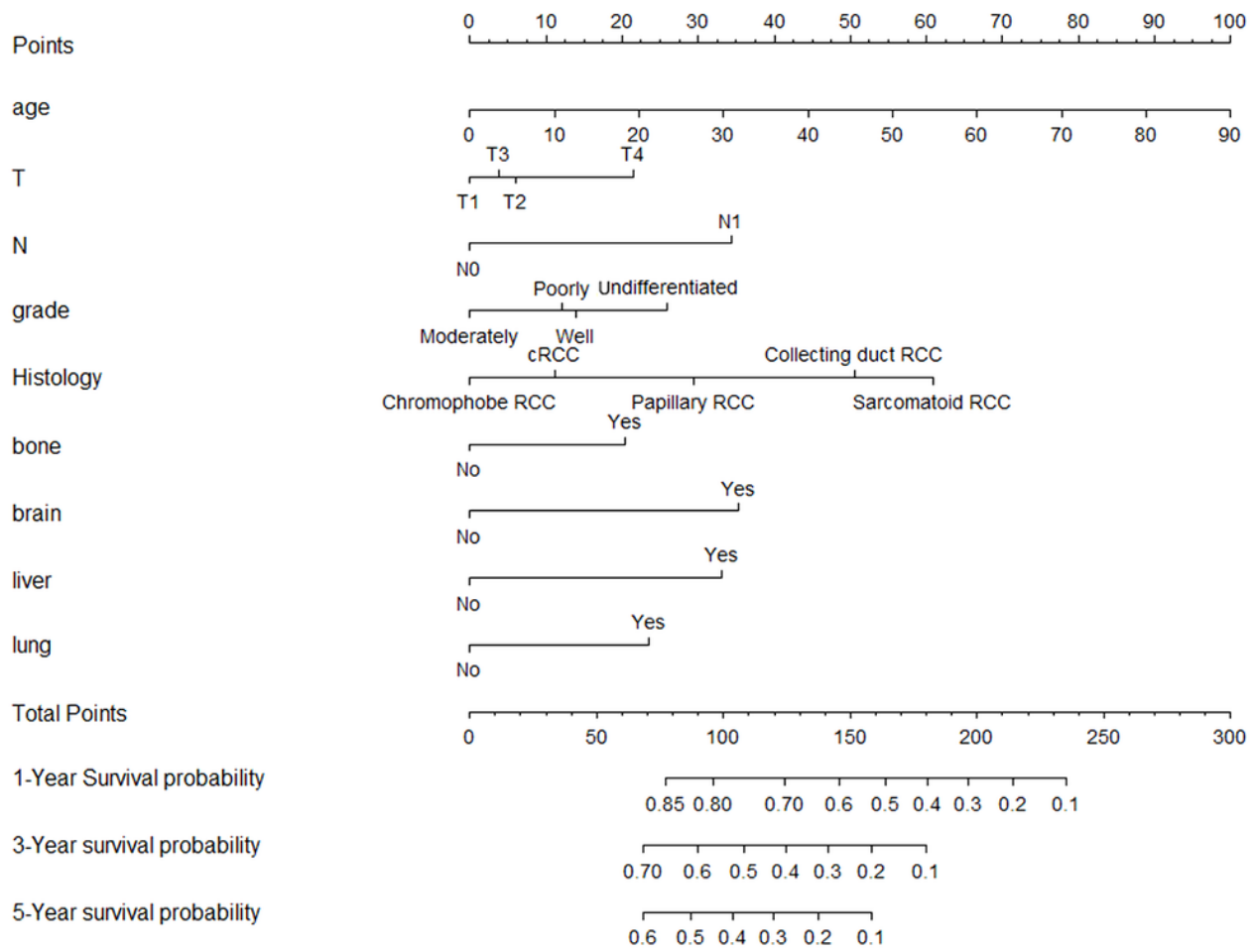

\section{Figure 4}

Nomogram for predicting the 1-year, 3-year and 5-year overall survival in patients with primary metastatic renal cell carcinoma. To obtain the predicted survival probability in meters, locate patient values on each axis. Based on the points line to acquire the number of points to add. Sum the points of all variables to determine the total point. A vertical line can be drawed down to the 1-year, 3-year and 5-year overall survival probability 